\title{
Analysis of an Adaptive Mixing Control Scheme for an Airbreathing Hypersonic Vehicle Model
}

\author{
Matthew Kuipers, Petros Ioannou, Barış Fidan, and Maj Mirmirani
}

\begin{abstract}
Recently the adaptive mixing control (AMC) approach has been applied to an airbreathing hypersonic flight vehicle (AHFV) model, and simulation results demonstrate that this adaptive scheme may be capable of improving performance when compared to a non-adaptive mixed- $\mu$ design with similar objectives. In this note, the analysis of this AMC scheme's stability and robustness properties is presented, establishing that if the unmodeled dynamics satisfy a norm bound condition, then the closed-loop states are bounded and the mean-square regulation error is of the order of the modeling error.
\end{abstract}

\section{INTRODUCTION}

The purpose of this paper is to present the stability and robustness analysis of an adaptive mixing control (AMC) scheme that was recently proposed in [1] for the control of an airbreathing hypersonic flight vehicle (AHFV) model that possesses significant complex and real uncertainties. AMC, as shown in Fig. 1, is a multiple model adaptive control (MMAC) approach that allows the designer to combine powerful modern multivariable tools (e.g., $\mathcal{H}_{\infty}$ and mixed$\mu$ synthesis) with online parameter estimation techniques of conventional robust adaptive control [2], [3]. As the simulation results of [1] demonstrate, the AMC scheme achieves a high-level of performance by adaptively utilizing a set of candidate controllers $K^{1}, \ldots, K^{8}$ that were constructed off line and each tuned to a small set of parameter uncertainty. The robust supervisor mixes these candidate controllers into the loop based on an online estimate of the unknown parameters. Because of space limitation, the interested reader is referred to [1] for a detailed presentation of the AMC design.

Notation: If $y: \mathbb{R}^{+} \rightarrow \mathbb{R}^{n}$, then the $\mathcal{L}_{p}$ norm of $y$ is denoted as $\|y\|_{p}$ and the truncated $\mathcal{L}_{2 \delta}$ norm is defined as

$$
\left\|y_{t}\right\|_{2 \delta} \triangleq\left(\int_{0}^{t} e^{-\delta(t-\tau)} y^{T}(\tau) y(\tau) d \tau\right)^{\frac{1}{2}}
$$

where $\delta \geq 0$ is a constant, provided that the integral in (1) exists. By $\left\|y_{t}\right\|_{2}$ we mean that $\left\|y_{t}\right\|_{2 \delta}$ with $\delta=0$, and we say that $y \in \mathcal{L}_{2 e}$ if $\left\|y_{t}\right\|_{2}$ exists. Let $y \in \mathcal{L}_{2 e}$, and consider the set

$$
\mathcal{S}(\mu)=\left\{y: \int_{t}^{t+T}|y(\tau)|^{2} d \tau \leq c_{0} \mu T+c_{1}, \forall t, T \geq 0\right\}
$$

M. Kuipers, who is the corresponding author, and P. Ioannou are with the Department of Electrical Engineering, University of Southern California. Contact: kuipers@usc. edu, ioannoudusc.edu

B. Fidan is with National ICT Australia Ltd. and Australian National University, Canberra, ACT 2601, Australia

M. Mirmirani is with Embry-Riddle Aeronautical University, Daytona Beach, FL, 32114

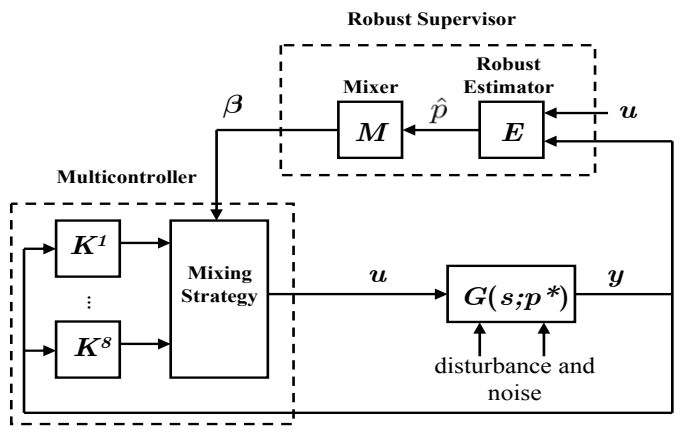

Fig. 1. Conceptual AMC architecture.

for a given constant $\mu$, where $c_{0}, c_{1} \geq 0$ are some finite constants, and $c_{0}$ and $c_{1}$ are independent of $\mu$. We say that $y$ is $\mu$-small in the mean square sense (m.s.s.) if $y \in \mathcal{S}(\mu)$. Furthermore, consider the signal $w:[0, \infty) \rightarrow \mathbb{R}^{+}$and the set

$$
\begin{aligned}
\mathcal{S}(w)= & \left\{y: \int_{t}^{t+T}|y(\tau)|^{2} d \tau\right. \\
& \left.\leq c_{0} \int_{t}^{t+T} w(\tau) d \tau+c_{1}, \quad \forall t, T \geq 0\right\}
\end{aligned}
$$

where $c_{0}, c_{1} \geq 0$ are some finite constants. We say that $y$ is $w$-small in the m.s.s. if $y \in \mathcal{S}(w)$. Let $H(s)$ and $h(t)$ be the transfer function and impulse response, respectively, of some linear time-invariant (LTI) system. If $H(s)$ is a proper transfer function and analytic in $\mathcal{R} e[s] \geq-\delta / 2$ for some $\delta>0$, where $\mathcal{R} e[s]$ denotes the real part of $s$, then the $\mathcal{H}_{\infty}$ system norm is given by $\|H\|_{\infty} \triangleq \sup _{j \omega}|H(j \omega)|$. The $\|\cdot\|_{2 \delta}$ system norm of $H(s)$ is defined as $\|H\|_{2 \delta} \triangleq$ $\frac{1}{\sqrt{2 \pi}}\left\{\int_{-\infty}^{\infty}\left|H\left(j \omega-\frac{\delta}{2}\right)\right|^{2} d \omega\right\}^{\frac{1}{2}}$. The induced $\mathcal{L}_{\infty}$ system norm of $H$ is given by $\|H\|_{i, \infty}=\|h\|_{1}$. If $y=H(s) u$ and $\|u\|_{\infty}=u_{0}$ then $|y(t)| \leq\|H\|_{i, \infty} u_{0}$ for all $t \geq 0$.

\section{Closed-Loop System}

We present the key equations used for analysis.

\section{A. Hypersonic Aircraft Model}

The AHFV considered in this paper is the computational fluid dynamics (CFD) based model developed in [4]. The linearized model is generated from the nonlinear equations of motion, which assumes a round non-rotating earth. The CFD approach captures the strong aero-propulsion interactions. Furthermore, the work [1] details the inclusion of 
complex uncertainty, manifesting from difficulties in modeling the non-stationary structural dynamics, and parametric uncertainty in the stability derivative $C_{M \alpha}$ and the control derivatives $C_{T \delta T}$ and $C_{M \delta e}$. The linearized AHFV dynamics are given by

$$
\begin{aligned}
& \dot{x}_{\mathbb{P}}=A_{\mathbb{P}}\left(p^{*}\right) x_{\mathbb{P}}+B_{\mathbb{P}}\left(p^{*}\right) u+\underbrace{B_{w} d_{w}+B_{s d} T_{s d}^{*}(s) \delta_{e}}_{\tilde{\eta}} \\
& u=G_{a c t}(s) u_{c}
\end{aligned}
$$

where $x_{\mathbb{P}}=\left[\begin{array}{llll}V & \gamma & h & \alpha\end{array}\right]^{T}$ is the AHFV state vector; $u=$ $\left[\begin{array}{ll}\delta_{T} & \delta_{e}\end{array}\right]^{T}$ is the control vector comprising throttle setting $\delta_{T}$ and elevon deflection $\delta_{e} ; u_{c}$ is the commanded control vector; $G_{a c t}$ is a stable transfer matrix representing actuator dynamics; $p^{*}$ is a vector of the unknown system parameters; $d_{w}=\left[\begin{array}{ll}d_{w, 1} & d_{w, 1}\end{array}\right]^{T}$ is a bounded atmospheric disturbance, i.e., $\left|d_{w, 1}(t)\right| \leq d_{1}$ for some $d_{1} \geq 0$ and $\left|d_{w, 2}(t)\right| \leq d_{2}$ for some $d_{2} \geq 0 ; T_{s d}^{*}$ is a stable rational transfer function that represents the structural dynamics. We introduce the notation $\tilde{\eta}$ to denote the modeling error term. The structural dynamics $T_{s d}^{*}$ are highly uncertain and, therefore, will be considered as unmodeled dynamics. We assume that $T_{s d}^{*}$ is analytic in $\mathcal{R} e[s] \geq-\delta_{0} / 2$ for some known $\delta_{0}>0$. Uncertainty in the parameters $a_{54}^{*}, b_{52}^{*}$, and $b_{11}^{*}$, where $a_{i j}^{*}$ and $b_{i j}^{*}$ denote the $i, j$ th component of $A_{\mathbb{P}}$ and $B_{\mathbb{P}}$, respectively, arise from the uncertainties in $C_{M \alpha}$, and $C_{M \delta e}$, and $C_{T \delta T}$. Thus, the parameter vector is given by $p^{*}=\left[\begin{array}{lll}a_{54}^{*} & b_{52}^{*} & b_{11}^{*}\end{array}\right]^{T}$ and each component is nonnegative. We consider $50 \%$ multiplicative uncertainty, i.e.,

$$
\begin{aligned}
p^{*} \in \Omega \triangleq\{p= & {\left[\begin{array}{lll}
p_{1} & p_{2} & p_{3}
\end{array}\right]^{T} \in \mathbb{R}^{3} } \\
& \left.: 0.5 p_{0 i} \leq p_{i} \leq 1.5 p_{0 i}, \quad i=1,2,3\right\}
\end{aligned}
$$

where $p_{0}=\left[\begin{array}{lll}p_{01} & p_{02} & p_{03}\end{array}\right]^{T}$ is the nominal value of $p^{*}$. The pair $\left(A_{\mathbb{P}}\left(p^{*}\right), B_{\mathbb{P}}\left(p^{*}\right)\right)$ is controllable for all $p^{*} \in \Omega$. Including the unmolded dynamics $T_{s d}^{*}$, the minimal state space realization of aircraft model of (2) has the form

$$
\begin{aligned}
& \dot{\bar{x}}_{\mathbb{P A}}=\bar{A}_{\mathbb{P A}}\left(p^{*}\right) \bar{x}_{\mathbb{P A}}+\bar{B}_{\mathbb{P A}}\left(p^{*}\right) u_{c}+\bar{B}_{w} d_{w} \\
& y=\bar{C}_{\mathbb{P A}} x_{\mathbb{P A}}, \quad C_{\mathbb{P A}}=\left[\begin{array}{ll}
I_{5 \times 5} & 0_{5 \times 4}
\end{array}\right]
\end{aligned}
$$

where

$$
\bar{x}_{\mathbb{P A}}(t)=\left[x_{\mathbb{P}}^{T}(t) u^{T}(t) x_{s d}^{T}(t)\right]^{T} \in \mathbb{R}^{\bar{n}}
$$

comprises the modeled state $x_{\mathbb{P}}$, the actuator state $u$, and the generalized elastic state $x_{s d}$, i.e., the state corresponding to $T_{s d}^{*}$. Neglecting $T_{s d}^{*}$, the transfer matrix $G\left(s ; p^{*}\right)$ denotes the modeled plant $G\left(s ; p^{*}\right)=\left(s I-A_{\mathbb{P}}\left(p^{*}\right)\right)^{-1} B_{\mathbb{P}}\left(p^{*}\right) G_{a c t}(s)$, and its realization

$$
\begin{aligned}
& \dot{x}_{\mathbb{P A}}=A_{\mathbb{P A}}\left(p^{*}\right) x_{\mathbb{P A}}+B_{\mathbb{P A}}\left(p^{*}\right) u_{c}, \quad x_{\mathbb{P A}} \triangleq\left[\begin{array}{ll}
x_{\mathbb{P}}^{T} & u^{T}
\end{array}\right]^{T} \\
& y=C_{\mathbb{P A}} x_{\mathbb{P} \mathbb{A}}, \quad C_{\mathbb{P A}}=\left[\begin{array}{ll}
I_{5 \times 5} & 0_{5 \times 2}
\end{array}\right] .
\end{aligned}
$$

\section{B. Adaptive Mixing Control Scheme}

The paper [1] presents the design of the AMC controller. Below we summarize the key equations and results relevant to its stability and robustness analysis.

Table I summarizes partition $\left\{\Omega^{1}, \ldots, \Omega^{8}\right\}$ of $\Omega$ used for the design of the candidate controller set $\left\{K^{1}, \ldots, K^{8}\right\}$.
TABLE I

Summary of PARAMETER PARTITIONING For CANDIDATE Controller Design

\begin{tabular}{c||cc|cc|cc}
\multicolumn{1}{l||}{} & \multicolumn{2}{|c|}{$a_{54}^{*}$} & \multicolumn{2}{c|}{$b_{52}^{*}$} & \multicolumn{2}{c}{$b_{11}^{*}$} \\
\hline \hline$\Omega^{1}$ & 3.15 & 6.78 & 2.55 & 5.49 & 47.07 & 101.27 \\
$\Omega^{2}$ & 3.15 & 6.78 & 2.55 & 5.49 & 91.63 & 141.22 \\
$\Omega^{3}$ & 3.15 & 6.78 & 4.97 & 7.66 & 47.07 & 101.27 \\
$\Omega^{4}$ & 3.15 & 6.78 & 4.97 & 7.66 & 91.63 & 141.22 \\
$\Omega^{5}$ & 6.13 & 9.45 & 2.55 & 5.49 & 47.07 & 101.27 \\
$\Omega^{6}$ & 6.13 & 9.45 & 2.55 & 5.49 & 91.63 & 141.22 \\
$\Omega^{7}$ & 6.13 & 9.45 & 4.97 & 7.66 & 47.07 & 101.27 \\
$\Omega^{8}$ & 6.13 & 9.45 & 4.97 & 7.66 & 91.63 & 141.22 \\
\hline \hline
\end{tabular}

Each candidate controller $K^{i}$ is designed to stabilize the plant if $p^{*} \in \Omega^{i}$. Moreover, the partition is such that $\Omega \subset$ $\cup_{i=1}^{8} \Omega^{i}$, ensuring that for any $p^{*} \in \Omega$ there exists a candidate controller that stabilizes the plant, and was designed to satisfy an overlapping property: for any parameter subset $\Omega^{i}$, each point belonging simultaneously to the boundary of $\Omega^{i}$ and to the interior of $\Omega$ also belongs to the interior of some parameter subset $\Omega^{j}$, where $i \neq j$. These overlapping regions provide a domain for control mixing.

The robust supervisor processes $(u, y)$ and outputs the mixing signal $\beta=\left[\begin{array}{llll}\beta^{1} & \ldots & \beta^{8}\end{array}\right]^{T}$, which determines the participation level of each controller. Thus, we define the set of all admissible mixing values at $p$ as

$$
\mathcal{B}_{p} \triangleq\left\{\beta \in[0,1]^{8}: \sum_{i=1}^{8} \beta^{i}=1 ; \beta^{i}=0, p \notin \Omega^{i}\right\} .
$$

Because $p^{*}$ is not known nor measurable, we cannot use $\mathcal{B}_{p^{*}}$ to define the mixing signal. The approach of AMC is to generate an online, recursive estimate $\hat{p}(t)$ of $p^{*}$, and, based based on certainty equivalence, generate a mixing signal $\beta(t) \in \mathcal{B}_{\hat{p}(t)}$ at every $t$. In [1], the mixer was constructed such that that the following properties hold:

M1 $\forall \hat{p} \in \Omega, \beta(\hat{p}) \in \mathcal{B}_{\hat{p}}$

M2 $\beta(\hat{p})$ is Lipschitz with respect to $\hat{p}$.

We now describe the robust online estimator. Let $\hat{p}_{12}(t)$ denote a vector comprising the estimates of $a_{54}^{*}$ and $b_{52}^{*}$; and $\hat{p}_{3}(t)$ denotes the estimate of $b_{11}^{*}$. Following the approach of [2], the gradient-method-based robust adaptive law is given by

$$
\begin{aligned}
\dot{\hat{p}}_{12} & =\operatorname{Pr}\left\{\Gamma_{12} \epsilon_{p 12} \phi_{12}\right\}, \quad \Gamma_{12}=\operatorname{diag}\left(10^{9}, 10^{11}\right) \\
\dot{\hat{p}}_{3} & =\operatorname{Pr}_{\bar{\Omega}_{3}}\left\{\Gamma_{3} \epsilon_{p 3} \phi_{3}\right\}, \quad \Gamma_{3}=10^{9}
\end{aligned}
$$

where

$$
\begin{aligned}
\epsilon_{p 12}= & \frac{z_{12}-\hat{p}_{12}^{T} \phi_{12}}{m^{2}}, \quad \epsilon_{p 3}=\frac{z_{3}-\hat{p}_{3}^{T} \phi_{3}}{m^{2}} \\
m^{2}= & 1+n_{d}, \quad \dot{n}_{d}=-\delta_{0} n_{d}+\delta_{e}^{2}, \quad \delta_{0}=0.4 \\
\bar{\Omega}_{12}= & \left\{\left(a_{54}, \quad b_{52}\right) \in \mathbb{R}^{2}\right. \\
& \left.\quad: 3.15 \leq a_{54} \leq 9.45, \quad 2.55 \leq b_{52} \leq 7.66\right\} \\
\bar{\Omega}_{3}= & \left\{b_{11} \in \mathbb{R}: 47.07 \leq b_{11} \leq 141.22\right\}
\end{aligned}
$$


$\operatorname{Pr}\{\cdot\}$ denotes the projection operator (cf. [3, Appendix A.10.3]), which is used to restrict the estimate to $\Omega$; and the auxiliary signals $z_{12}(t), z_{3}(t), \phi_{12}(t), \phi_{3}(t)$ are generated by the filters

$$
\begin{aligned}
z_{12} & =\frac{s}{\Lambda(s)} q-\frac{1}{\Lambda(s)}\left(\sum_{j \in\{1,2,3,5\}} a_{5 j} x_{j}+b_{51} \delta_{T}\right) \\
z_{3} & =\frac{s}{\Lambda(s)} V-\frac{1}{\Lambda(s)}\left(\sum_{j=1}^{5} a_{1 j} x_{j}+b_{12} \delta_{e}\right) \\
\phi_{12} & =\frac{1}{\Lambda(s)}\left[\begin{array}{c}
\alpha \\
\delta_{e}
\end{array}\right], \quad \phi_{3}=\frac{1}{\Lambda(s)} \delta_{T}, \quad \Lambda(s)=(s+100)^{2}
\end{aligned}
$$

where $x_{i}$ denotes the $i$ th component of $x_{\mathbb{P}}$, or equivalently $y$. The design constant $\delta_{0}>0$ was chosen such that $T_{s d}^{*}$ is analytic in $\mathcal{R} e[s] \geq-\delta_{0} / 2$. Let the filters of (15)-(17) be given a minimal realization of the form

$$
\begin{aligned}
& \dot{x}_{\mathbb{E}}=A_{\mathbb{E}} x_{\mathbb{E}}+B_{\mathbb{E}}\left[\begin{array}{ll}
y & u
\end{array}\right]^{T} \\
& z_{12}=C_{z 1} x_{\mathbb{E}}, \quad \phi_{12}=C_{\phi 1} x_{\mathbb{E}} \\
& z_{3}=C_{z 2} x_{\mathbb{E}}, \quad \phi_{3}=C_{\phi 2} x_{\mathbb{E}} .
\end{aligned}
$$

The adaptive law (8)-(9) guarantees the following properties

$$
\begin{aligned}
\epsilon_{p 12}, \epsilon_{p 12} m, \dot{\hat{p}}_{12} & \in \mathcal{S}\left(\eta_{12} / m^{2}\right) \cap \mathcal{L}_{\infty} \\
\epsilon_{p 3}, \epsilon_{p 3} m, \dot{\hat{p}}_{3} & \in \mathcal{S}\left(\eta_{3} / m^{2}\right) \cap \mathcal{L}_{\infty} \\
\hat{p}_{12}(t) & \in \bar{\Omega}_{12}, \quad \hat{p}_{3}(t) \in \bar{\Omega}_{3}, \quad \forall t \geq 0
\end{aligned}
$$

where

$$
\begin{aligned}
\eta_{12} & =\frac{1}{\Lambda(s)}\left(\left[\begin{array}{ll}
b_{w, 51} & b_{w, 52}
\end{array}\right] d_{w}+b_{s d, 5} T_{s d}^{*} \delta_{e}\right) \\
\eta_{3} & =\frac{1}{\Lambda(s)}\left(\left[\begin{array}{ll}
b_{w, 11} & b_{w, 12}
\end{array}\right] d_{w}+b_{s d, 1} T_{s d}^{*} \delta_{e}\right) .
\end{aligned}
$$

where $b_{w, i j}$ denotes the $i j$ th component of $B_{w}$.

We now describe the multicontroller and how the candidate controllers are mixed stably. Let $K_{0}$ be a stabilizing controller for any $p^{*} \in \Omega$. Consider the co-prime factorizations of $G\left(s ; p_{0}\right)$ and $K_{0}$

$$
\begin{array}{cc}
G=N M^{-1}=\tilde{M}^{-1} \tilde{N}, & N, M, \tilde{N}, \tilde{M} \in \mathcal{R} \mathcal{H}_{\infty} \\
K_{0}=U_{0} V_{0}^{-1}=\tilde{V}_{0}^{-1} \tilde{U}_{0}, & U_{0}, V_{0}, \tilde{U}_{0}, \tilde{V}_{0} \in \mathcal{R} \mathcal{H}_{\infty}
\end{array}
$$

where $p_{0} \in \cap_{i=1}^{8} \Omega^{i} ; \mathcal{R} \mathcal{H}_{\infty}$ denotes the set of rational stable transfer functions; and the eight transfer matrices in (26)-(27) satisfy the double Bezout equation. Similarly, we consider the coprime factorizations of the candidate controllers

$$
\begin{aligned}
& K^{i}=U^{i}\left(V^{i}\right)^{-1}=\left(\tilde{V}^{i}\right)^{-1} \tilde{U}^{i} \\
& U^{i}, V^{i}, \tilde{U}^{i}, \tilde{V}^{i} \in \mathcal{R} \mathcal{H}_{\infty}, \quad i=1, \ldots, 8
\end{aligned}
$$

where the coprime factorizations of $K^{i}$ and $G$ satisfy the double Bezout equation.

The multicontroller $K(\beta)$ is given by

$$
K(\beta)=\mathcal{F}_{l}\left(J_{K_{0}}, Q(\beta)\right)
$$

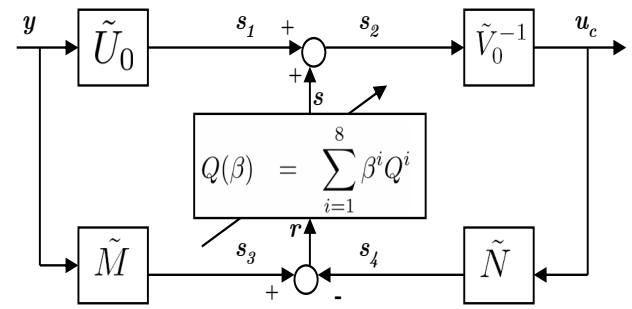

Fig. 2. Multicontroller implemented by $Q$-mixing

where

$$
\begin{aligned}
& J_{K_{0}}=\left[\begin{array}{cc}
U_{0} V_{0}^{-1} & \tilde{V}_{0}^{-1} \\
V_{0}^{-1} & -V_{0}^{-1} N
\end{array}\right]=\left[\begin{array}{cc}
\tilde{V}_{0} \tilde{U}_{0}^{-1} & \tilde{V}_{0}^{-1} \\
V_{0}^{-1} & -V_{0}^{-1} N
\end{array}\right] \\
& Q(\beta)=\sum_{i=1}^{8} \beta^{i} Q^{i}, \quad Q^{i}=\tilde{V}^{i}\left(K^{i}-K_{0}\right) V_{0}
\end{aligned}
$$

The reader is referred to [5] for details on deriving the stable filters $Q^{1}, \ldots, Q^{8}$ of (30) and the following result.

Theorem 1: Consider the plant $y=G\left(s ; p^{*}\right) u$ and the candidate controllers $K^{1}, \ldots, K^{8}$, of which at least one is stabilizing for any $p^{*} \in \Omega$. If $K(\beta)$ is given by (28), then $K\left(e_{i}\right)=K^{i}$, where $e_{i}$ is the $i$-th standard vector in $\mathbb{R}^{8}$. Additionally, if the control law is given by $u=K\left(\beta^{*}\right) y$, where $\beta^{*} \in \mathcal{B}_{p^{*}}$, then $K\left(\beta^{*}\right)$ the closed-loop system is output stabilizing.

The proof of this result follows from a straightforward interpretation of [5].

For internal stability, we must show that the multicontroller is detectable. The multicontroller $K(\beta)$ is implemented with the internal structure [6] shown in Fig. 2. Consider the minimal state-space realizations for $\tilde{U}_{0}, \tilde{V}_{0}^{-1}$, $\tilde{M}$, and $\tilde{N}$ given by

$$
\begin{aligned}
& \dot{x}_{\tilde{U}}=A_{\tilde{U}} x_{\tilde{U}}+B_{\tilde{U}} y, \quad s_{1}=C_{\tilde{U}} x_{\tilde{U}} \\
& \dot{x}_{\tilde{V}}=A_{\tilde{V}} x_{\tilde{V}}+B_{\tilde{V}} s_{2}, \quad u_{c}=C_{\tilde{V}} x_{\tilde{V}}+D_{\tilde{V}} s_{2} \\
& \dot{x}_{\tilde{M}}=A_{\tilde{M}} x_{\tilde{M}}+B_{\tilde{M}} y, \quad s_{3}=C_{\tilde{M}} x_{\tilde{M}}+D_{\tilde{M}} y \\
& \dot{x}_{\tilde{N}}=A_{\tilde{N}} x_{\tilde{N}}+B_{\tilde{N}} u_{c}, \quad s_{4}=C_{\tilde{N}} x_{\tilde{N}}
\end{aligned}
$$

respectively. Similarly, the eight filters $Q^{1}, \ldots, Q^{8}$ are given by the minimal realizations

$$
\dot{x}_{Q i}=A_{Q i} x_{Q i}+B_{Q i} r, \quad s=C_{Q i} x_{Q i}, \quad i=1, \ldots, 8
$$

and, from (30), we realize $Q(\beta)$ as

$$
\dot{x}_{Q}=A_{Q} x_{Q}+B_{Q} r, \quad s=C_{Q} x_{Q}
$$

where $A_{Q}=\operatorname{diag}\left(A_{Q 1}, \ldots, A_{Q 8}\right), \quad B_{Q}=$ $\left[\begin{array}{lll}B_{Q 1}^{T} & \ldots & B_{Q 8}^{T}\end{array}\right]^{T}$, and $C_{Q}=\left[\begin{array}{lll}\beta^{1} C_{Q 1} & \ldots & \beta^{8} C_{Q 8}\end{array}\right]$. The overall state-space realization for the multicontroller $K(\beta)$ is given by

$$
\dot{x}_{\mathbb{C}}=A_{\mathbb{C}}(\beta) x_{\mathbb{C}}+B_{\mathbb{C}} y, \quad u_{c}=C_{\mathbb{C}}(\beta) x_{\mathbb{C}}
$$


where the composite controller state is given by $x_{\mathbb{C}}=$ $\left[x_{\tilde{M}}^{T} x_{\tilde{U}}^{T} x_{\tilde{V}}^{T} x_{\tilde{N}}^{T} x_{Q}^{T}\right]^{T}$ and the system matrices are defined obviously. By implementing the multicontroller as in Fig. 2, the pair $\left(C_{\mathbb{C}}(\beta), A_{\mathbb{C}}(\beta)\right)$ is detectable for all $\beta \in \mathbb{R}^{8}$. A sketch of the proof is as follows: It can be shown that all unstable modes are detectable by establishing that if $y, u_{c} \rightarrow 0$ as $t \rightarrow \infty$, then $\lim _{t \rightarrow \infty} x_{\mathbb{C}}(t)=0$ for any initial condition $x_{\mathbb{C}}(0)$. So, suppose $y, u_{c} \rightarrow 0$. Then, from the stability of the filters $\tilde{U}_{0}, \tilde{M}$, and $\tilde{N}$, it follows that $s_{1}, s_{3}, s_{4} \rightarrow 0$. In turn, we have that $r \rightarrow 0$ and, because $Q$ is a stable filter, $s \rightarrow 0$. Thus, it follows from $s_{1}, s_{3}, s_{4}, u_{c} \rightarrow 0$ and the detectability of the realizations (31) - (36) that $x_{\tilde{U}}, x_{\tilde{V}}, x_{\tilde{M}}, x_{\tilde{N}} \rightarrow 0$ and, in turn, $x_{\mathbb{C}} \rightarrow 0$. Therefore, the realization (37) is detectable.

It follows from Theorem 1 and the definition of $\mathcal{B}_{p^{*}}$, if mixing signal $\beta(t)$ takes on the constant value $\beta^{*} \in \mathcal{B}_{p^{*}}$ for all $t$, then the LTI controller $K\left(\beta^{*}\right)$ stabilizes the plant $G\left(s ; p^{*}\right)$, i.e., the closed-loop system satisfies

$$
\left[\begin{array}{c}
\dot{x}_{\mathbb{P} \mathbb{A}} \\
\dot{x}_{\mathbb{C}}
\end{array}\right]=\left[\begin{array}{cc}
A_{\mathbb{P A}}\left(p^{*}\right) & B_{\mathbb{P A}}\left(p^{*}\right) C_{\mathbb{C}}\left(\beta^{*}\right) \\
B_{\mathbb{C}} & A_{\mathbb{C}}\left(\beta^{*}\right)
\end{array}\right]\left[\begin{array}{c}
x_{\mathbb{P A}} \\
x_{\mathbb{C}}
\end{array}\right]
$$

and, because $K\left(\beta^{*}\right)$ is stabilizing, $x_{\mathbb{P A}}, x_{\mathbb{C}} \rightarrow 0$ as $t \rightarrow$ $\infty$. It is important to note that this result is not sufficient for establishing stability of the closed-loop adaptive system. The stability and robustness analysis of the AMC scheme is presented in the sequel.

Combining the multicontroller $K(\beta)$, mixer $M$, and adaptive law yields the adaptive control law $u_{c}=K(\beta(\hat{p})) y$. The closed-loop states of the AMC scheme are $x \triangleq$ $\left[\begin{array}{lll}\bar{x}_{\mathbb{P} \mathbb{A}}^{T} & x_{\mathbb{C}}^{T} & x_{\mathbb{E}}^{T}\end{array}\right]^{T}, \hat{p} \triangleq\left[\begin{array}{ll}\hat{p}_{12}^{T} & \hat{p}_{3}\end{array}\right]^{T}$, and $n_{d}$.

\section{Stability And Robustness AnAlysis}

The following key results are used in the stability and robustness analysis of AMC schemes. The results are well known, and, unless stated otherwise, their proofs can be found in [2] and the references within.

Theorem 2: Let $\Omega \subset \mathbb{R}^{2 n}$ be compact and $\theta$ be any constant in $\Omega$. Let the parameterized detectible pair $(C(\theta), A(\theta))$ be Lipschitz with respect to $\theta \in \Omega$, where $A(\theta) \in \mathbb{R}^{n \times n}$ and $C(\theta) \in \mathbb{R}^{l \times n}$.

1) Then there exists a Lipschitz function $L: \Omega \rightarrow \mathbb{R}^{n \times l}$, such that $A_{I}(\theta) \triangleq A(\theta)-L(\theta) C(\theta)$ is a stability matrix uniformly in $\theta \in \Omega$, i.e., $A_{I}(\theta)$ satisfies

$$
\max _{i} \mathcal{R} e\left\{\lambda_{i}\left[A_{I}(\theta)\right]\right\}<-\sigma
$$

for some $\sigma>0$ independent of $\theta$, where $\lambda_{i}\left(A_{I}(\theta)\right)$ is the $i^{t h}$ eigenvalue of the matrix $A_{I}(\theta)$.

2) If $\theta(t) \in \Omega$ for all $t \geq 0$ and $\dot{\theta} \in \mathcal{L}_{2}$ is satisfied in addition to the conditions in 1), then the equilibrium $x_{e}=0$ of $\dot{x}=A_{I}(\theta(t)) x$ is e.s.

3) If $\theta(t) \in \Omega$ for all $t \geq 0$ and $\dot{\theta} \in \mathcal{S}\left(\mu^{2}\right)$ is satisfied in addition to the conditions in 1), then there exists a $\mu^{*}>0$ such that if $\mu \in\left[0, \mu^{*}\right)$ the equilibrium $x_{e}=0$ of $\dot{x}=A_{I}(\theta(t)) x$ is e.s.
The proof of Theorem 2 is a combination of the well-known results of [7] and the linear time varying (LTV) stability results found in [2].

The following result concerns the LTV system given by

$$
\begin{aligned}
\dot{x} & =A(t) x+B(t) u, \quad x(0)=x_{0} \\
y & =C(t) x+D(t) u
\end{aligned}
$$

where $x(t) \in \mathbb{R}^{n}, y(t) \in \mathbb{R}^{l}, u(t) \in \mathbb{R}^{m}$, and the elements of the matrices $A(t) \in \mathbb{R}^{n \times n}, B(t) \in \mathbb{R}^{n \times m}, C(t) \in \mathbb{R}^{l \times n}$, and $D(t) \in \mathbb{R}^{l \times m}$ are bounded continuous functions of time.

Lemma 1: If the LTV system (40),(41) is e.s. and $u \in \mathcal{L}_{2 e}$ then

1) for any $\delta \in\left[0, \delta_{1}\right)$ where $0<\delta_{1}<2 \alpha_{0}$ is arbitrary, we have

$$
\left\|x_{t}\right\|_{2 \delta} \leq \frac{c \lambda_{0}}{\sqrt{\left(\delta_{1}-\delta\right)\left(2 \alpha_{0}-\delta\right)}}\left\|u_{t}\right\|_{2 \delta}+\epsilon_{t}
$$

where $c=\sup _{t}\|B\|$ and $\epsilon_{t}$ is an exponentially decaying to zero term because $x_{0} \neq 0$.

2) $u \in \mathcal{L}_{2} \Rightarrow x \in \mathcal{L}_{2} \cap \mathcal{L}_{\infty}, \dot{x} \in \mathcal{L}_{2}$, and $\lim _{t \rightarrow \infty}|x(t)|=$ 0

3) $u \in \mathcal{S}(\mu) \Rightarrow x \in \mathcal{S}(\mu) \cap \mathcal{L}_{\infty}$

Lemma 2: Consider the LTI system given by $y=H(s) u$ where $H(s)$ is a strictly proper rational function of $s$. If $H(s)$ is analytic in $\mathcal{R} e[s] \geq-\delta / 2$ for some $\delta \geq 0$ and $u \in \mathcal{L}_{2 e}$ then we have $|y(t)| \leq\|H(s)\|_{2 \delta}\left\|u_{t}\right\|_{2 \delta}$.

The following Bellman-Gronwall (B-G) lemma is useful for establishing boundedness.

Lemma 3 (B-G Lemma): Let $c_{1}, c_{2}$ be positive constants and $g(t)$ be a piece-wise continuous function of $t$. If for all $t \geq t_{0} \geq 0$, the function $y(t)$ satisfies the inequality

$$
y(t) \leq c_{1}+c_{2} \int_{t_{0}}^{t} e^{-\delta(t-\tau)} g^{2}(\tau) y(\tau) d \tau
$$

then for all $t \geq t_{0} \geq 0$

$$
\begin{aligned}
y(t) \leq & c_{1} e^{-\delta\left(t-t_{0}\right)} e^{c_{2} \int_{t_{0}}^{t} g^{2}(\tau) d \tau} \\
& +c_{1} \delta \int_{t_{0}}^{t} e^{-\delta(t-s)} e^{c_{2} \int_{s}^{t} g^{2}(\tau) d \tau} d s .
\end{aligned}
$$

Theorem 3: Consider the unknown plant given by (4), with $d_{w}$ bounded. The AMC scheme comprising the multicontroller (37), a mixer that satisfies M1 and M2, and the robust online parameter estimators (8) and (9) guarantees that

1) there exists a constant $\delta^{*}>0$ such that if

$$
\begin{aligned}
& c\left(\Delta_{11}^{2}+\Delta_{21}^{2}\right)<\delta^{*} \\
& \Delta_{11} \triangleq\left|b_{s d, 5}\right|\left\|\frac{1}{\Lambda(s)} T_{s d}^{*}\right\|_{2 \delta_{0}} \\
& \Delta_{21} \triangleq\left|b_{s d, 1}\right|\left\|\frac{1}{\Lambda(s)} T_{s d}^{*}\right\|_{2 \delta_{0}}
\end{aligned}
$$

where $c$ is a finite constant, then $x, \hat{p}, n_{d} \in \mathcal{L}_{\infty}$ and $\int_{0}^{t}|y(\tau)|^{2} d \tau \leq c\left(\Delta_{11}^{2}+\Delta_{21}^{2}+\left\|d_{w}\right\|_{\infty}^{2}\right) t+c$.

2) if $T_{s d}^{*}, d_{w}=0$, then $x \rightarrow 0$ as $t \rightarrow \infty$. 
Proof: Let us rewrite (4), (37), and (18) as

$$
\begin{aligned}
& \dot{x}=A(\hat{p}) x+B d_{w}, \quad x \triangleq\left[\begin{array}{llll}
\bar{x}_{\mathbb{P}}^{T} & u^{T} & x_{\mathbb{C}}^{T} & x_{\mathbb{E}}^{T}
\end{array}\right]^{T} \\
& \epsilon_{1}=C(\hat{p}) x, \quad \epsilon_{1} \triangleq\left[z_{12}-\hat{p}_{12}^{T} \phi_{12} \quad z_{3}-\hat{p}_{3} \phi_{3}\right]^{T}
\end{aligned}
$$

where the matrices $A(\hat{p}), B$, and $C(\hat{p})$ are defined obviously, and the unnormalized estimation error $\epsilon_{1}$ drives the adaptive laws (8) and (9), which tune $\hat{p}(t)$. The closed-loop system written in the form of (45)-(46) is suitable for the application of Morse's tunability analysis [8] approach. Consider the arbitrary initializations $x(0)=x_{0}$ and $\hat{p}(0)=p_{0} \in \Omega$. It has been establish in [9] that there exists a unique global solution $\left[\begin{array}{lll}x^{T}(t) & \hat{p}^{T}(t) n_{d}(t)\end{array}\right]^{T}$ along the trajectories of (8), (9), (11), and (45), $\forall t \in[0, \infty)$.

Step 1: Establish that $\forall \hat{p} \in \Omega,\{C(\hat{p}), A(\hat{p})\}$ is a detectible pair.

If we let $\epsilon_{1} \equiv 0$ then there is no adaptation, i.e., $\hat{p} \equiv$ $p_{0}=\left[\begin{array}{ll}p_{12,0}^{T} & p_{3,0}\end{array}\right]^{T}=\left[\begin{array}{lll}a_{54,0} & b_{52,0} & b_{11,0}\end{array}\right]^{T}$, where $a_{54,0}$, $b_{52,0}$, and $b_{11,0}$ are the initial estimates of $a_{54}, b_{52}$, and $b_{11}$, respectively. Therefore, the closed-loop system is an LTI system.

Because $\epsilon_{1} \equiv 0$, we have that $z_{12}=p_{12,0}^{T} \phi_{12}$ and $z_{3}=$ $p_{3,0} \phi_{3}$. For the case of $z_{12}=p_{12,0}^{T} \phi_{12}$, it follows from (15) and (17) that $x_{\mathbb{P}}$ and $u$ satisfy

$$
\begin{array}{r}
\frac{s}{\Lambda(s)} q=\frac{1}{\Lambda(s)}\left(a_{51} V+a_{52} \gamma+a_{53} h+a_{54,0} \alpha\right. \\
\left.+a_{55} q+b_{51} \delta_{T}+b_{52,0} \delta_{e}\right)
\end{array}
$$

or, equivalently,

$$
\begin{aligned}
\dot{q}=a_{51} V & +a_{52} \gamma+a_{53} h+a_{54,0} \alpha \\
& +a_{55} q+b_{51} \delta_{T}+b_{52,0} \delta_{e} .
\end{aligned}
$$

Furthermore, from $z_{3}=p_{3,0} \phi_{3}$, (16), and (17), $x_{\mathbb{P}}$ and $u$ also satisfy

$$
\begin{array}{r}
\frac{s}{\Lambda(s)} V=\frac{1}{\Lambda(s)}\left(a_{11} V+a_{12} \gamma+a_{13} h+a_{14} \alpha\right. \\
\left.+a_{15} q+b_{11,0} \delta_{T}+b_{12} \delta_{e}\right)
\end{array}
$$

or, equivalently,

$$
\begin{aligned}
\dot{V}=a_{11} V & +a_{12} \gamma+a_{13} h+a_{14} \alpha \\
& +a_{15} q+b_{11,0} \delta_{T}+b_{12} \delta_{e} .
\end{aligned}
$$

Therefore by combining (48) and (50) with the state equations for $h, \gamma$, and $\alpha$, as well as the actuator model, we have that

$$
\dot{x}_{\mathbb{P} \mathbb{A}}=A_{\mathbb{P} \mathbb{A}}\left(p_{0}\right) x_{\mathbb{P A}}+B_{\mathbb{P A}}\left(p_{0}\right) u_{c}, \quad x_{\mathbb{P A}} \triangleq\left[\begin{array}{ll}
x_{\mathbb{P}}^{T} & u^{T}
\end{array}\right]^{T}
$$

where $A_{\mathbb{A} \mathbb{A}}$ and $B_{\mathbb{P A}}$ are defined obviously. Since $\hat{p} \equiv p_{0}$, the multicontroller is the LTI system

$$
\begin{aligned}
\dot{x}_{\mathbb{C}} & =A_{\mathbb{C}}\left(\beta\left(p_{0}\right)\right) x_{\mathbb{C}}+B_{\mathbb{C}} y \\
u_{c} & =C_{\mathbb{C}}\left(\beta\left(p_{0}\right)\right) x_{\mathbb{C}} .
\end{aligned}
$$

By combining (51) and (52), we obtain the requirement that $x_{\mathbb{P}}$ and $u$ satisfies

$$
\left[\begin{array}{c}
\dot{x}_{\mathbb{P} \mathbb{A}} \\
\dot{x}_{\mathbb{C}}
\end{array}\right]=\left[\begin{array}{cc}
A_{\mathbb{A} A}\left(p_{0}\right) & B_{\mathbb{P A}}\left(p_{0}\right) C_{\mathbb{C}}\left(\beta\left(p_{0}\right)\right) \\
B_{\mathbb{C}} & A_{\mathbb{C}}\left(\beta\left(p_{0}\right)\right)
\end{array}\right]\left[\begin{array}{c}
x_{\mathbb{P A}} \\
x_{\mathbb{C}}
\end{array}\right] .
$$

It then follows from the design of the multicontroller and mixer as discussed in Section II that $x_{\mathbb{A} \mathbb{A}}, x_{\mathbb{C}} \rightarrow 0$, and in turn $\bar{x}_{\mathbb{P}}, x_{\mathbb{E}} \rightarrow 0$. Therefore, the pair $\left(C\left(p_{0}\right), A\left(p_{0}\right)\right)$ is detectable.

Step 2: Establish that along the solutions of (8), (11), (9), and (45) there exists a function $L: \Omega \rightarrow \mathbb{R}^{\bar{n} \times \bar{n}}$ such that $A_{I}(t) \triangleq A(\hat{p}(t))-L(\hat{p}(t)) C(\hat{p}(t))$ is exponentially stable.

We define the normalized estimation error as $\epsilon \triangleq$ $\left[\begin{array}{ll}\epsilon_{p 12} & \epsilon_{p 3}\end{array}\right]^{T}=\epsilon_{1} / m^{2}$ and the modeling error term as $\eta_{a} \triangleq\left[\begin{array}{ll}\eta_{12} & \eta_{3}\end{array}\right]^{T}$. From the properties (21)-(22), the robust adaptive law (8),(9) guarantees

$$
\epsilon, \epsilon m, \dot{\hat{p}} \in \mathcal{S}\left(\eta_{a}^{T} \eta_{a} / m^{2}\right) .
$$

Applying Lemma 2 to (24), together with $d_{w, 1} \leq d_{1}$ and $d_{w, 2} \leq d_{2}$, yields

$$
\left|\eta_{12}(t)\right| \leq \Delta_{11}\left\|\left(\delta_{e}\right)_{t}\right\|_{2 \delta_{0}}+\Delta_{12}
$$

where $\Delta_{11}$ is defined in (43) and $\Delta_{12}$ is some finite constant. Similarly, from (25) and the boundedness of $d_{w}(t)$, we obtain the bound

$$
\left|\eta_{3}(t)\right| \leq \Delta_{21}\left\|\left(\delta_{e}\right)_{t}\right\|_{2 \delta_{0}}+\Delta_{22}
$$

where $\Delta_{21}$ is defined in (43) and $\Delta_{22}$ is some finite constant. Since $m^{2}=1+\left\|\left(\delta_{e}\right)_{t}\right\|_{2 \delta_{0}}^{2}$ and $m \geq 0$ it follows that

$$
\frac{\left|\eta_{a}(t)\right|^{2}}{m^{2}} \leq \mu^{2} \triangleq c\left(\Delta_{11}^{2}+\Delta_{12}^{2}+\Delta_{21}^{2}+\Delta_{22}^{2}\right)
$$

for some constant $c>0$. Therefore, we have

$$
\epsilon, \epsilon m, \dot{\hat{p}} \in \mathcal{S}\left(\mu^{2}\right) \text {. }
$$

The matrix $A(\hat{p})$ depends on $\hat{p}$ as $A_{\mathbb{C}}(\beta(\hat{p}))$ and $C_{\mathbb{C}}(\beta(\hat{p}))$ depend on $\hat{p}$. $A_{\mathbb{C}}(\beta(\hat{p}))$ and $C_{\mathbb{C}}(\beta(\hat{p}))$ are linear in $\beta$, and $\beta$ is Lipschitz in $\hat{p}$. Therefore, $A$ is Lipschitz in $\hat{p}$. $C$ is affine in $\hat{p}$, and, therefore, also Lipschitz. Furthermore, because the adaptive law guarantees that $\hat{p}(t) \in \Omega$ and $\dot{\hat{p}} \in \mathcal{S}\left(\mu^{2}\right)$, it follows from the detectability result of Step 1 and result 3 ) of Theorem 2 that there exists a continuous function $L$ : $\Omega \rightarrow \mathbb{R}^{\bar{n} \times \bar{n}}$ such that that $A_{I}(t) \triangleq A(\hat{p}(t))-L(\hat{p}(t)) C(\hat{p}(t))$ is e.s., i.e., the transition matrix $\Phi(t, \tau)$ of $A_{I}(t)$ satisfies $\|\Phi(t, \tau)\| \leq \lambda_{0} e^{-\alpha_{0}(t-\tau)}$ for some positive constants $\lambda_{0}, \alpha_{0}$ and $t \geq \tau \geq 0$, provided that

$$
\mu^{2}<\mu^{*}
$$

for some $\mu^{*}>0$. Assume the filter $1 / \Lambda(s)$ is chosen so that $c\left(\Delta_{12}^{2}+\Delta_{22}^{2}\right)$ is sufficiently small, say $c\left(\Delta_{12}^{2}+\Delta_{22}^{2}\right)<$ $\mu^{*} / 2$ so that for $c\left(\Delta_{11}^{2}+\Delta_{21}^{2}\right)<\mu^{*} / 2$, condition (59) is always satisfied ${ }^{1}$. Note that if $T_{s d}^{*}, d_{w}=0$, the adaptive law

\footnotetext{
${ }^{1}$ Boundedness of the closed-loop signals can be proven independent of the size of $c\left(\Delta_{12}^{2}+\Delta_{22}^{2}\right)$ by using the analysis approach of [2, Section 9.9.1], which involves a complicated, lengthy contradiction argument. The analysis presented, however, has been chosen for simplicity.
} 
guarantees that $\dot{\theta} \in \mathcal{L}_{2}$, and from result 2) of Theorem 1, we have that $A_{I}(t)$ is e.s. Also observe that in either case, $\|L\| \in \mathcal{L}_{\infty}$ since $L$ is continuous and $\Omega$ is compact.

Step 3: Establish boundedness and convergence of $x$.

Let $\delta \in\left[0, \delta_{1}\right)$, where $\delta_{1}<\min \left\{2 \alpha_{0}, \delta_{0}\right\}$, and $c>0$ denotes any finite constant.

By applying output injection, we rewrite (45),(46) as

$$
\dot{x}=A_{I}(t) x+B d_{w}+L(\hat{p}(t)) \epsilon_{1}
$$

where in Step 2 we established e.s. of the homogeneous part of (60).

We establish that $m \in \mathcal{L}_{\infty}$ : By result 1) of Lemma 1 and the e.s. property of $A_{I}$, we have that

$$
\left\|x_{t}\right\|_{2 \delta} \leq c\left\|\left(\epsilon_{1}\right)_{t}\right\|_{2 \delta}+c .
$$

Applying the $\mathcal{L}_{2 \delta}$ norm to $u_{c}=C_{\mathbb{C}}(t) x_{\mathbb{C}}$, where $\left\|C_{\mathbb{C}}(t)\right\|$ is bounded (because $C_{\mathbb{C}}$ is Lipschitz in $\hat{p}$ and $\Omega$ is compact,) yields

$$
\left\|\left(\delta_{e}\right)_{t}\right\|_{2 \delta} \leq\left\|\left(u_{c}\right)_{t}\right\|_{2 \delta} \leq c\left\|\left(x_{\mathbb{C}}\right)_{t}\right\|_{2 \delta} \leq c\left\|\left(\epsilon_{1}\right)_{t}\right\|_{2 \delta}+c
$$

where the third inequality is obtained by first recognizing that $x_{\mathbb{C}}$ is a subvector of $x$ and then applying inequality (61). Consider the fictitious normalization signal

$$
m_{f}^{2} \triangleq 1+\left\|\left(\delta_{e}\right)_{t}\right\|_{2 \delta}^{2} .
$$

Note that because $\delta<\delta_{0}$, it follows from the definitions of $m, m_{f}$ that $m \leq m_{f}$. Substituting (62), and $\epsilon_{1}=\epsilon m^{2}$ into (63) yields

$$
m_{f}^{2} \leq c\left\|\left(\epsilon m^{2}\right)_{t}\right\|_{2 \delta}^{2}+c \leq c\left\|\left(\epsilon m m_{f}\right)_{t}\right\|_{2 \delta}^{2}+c
$$

where the second inequality is obtained by using $m \leq m_{f}$. From the definition of $\left\|(\cdot)_{t}\right\|_{2 \delta}$ it follows that

$$
m_{f}^{2} \leq c \int_{0}^{t} e^{-\delta(t-\tau)}(\epsilon(\tau) m(\tau))^{2} m_{f}^{2}(\tau) d \tau+c .
$$

Applying the B-G Lemma to (65) with $g(\tau)=\epsilon(\tau) m(\tau)$ yields

$$
m_{f}^{2} \leq c e^{-\delta t} e^{c \int_{0}^{t} g^{2}(\tau) d \tau}+c \delta \int_{0}^{t} e^{-\delta(t-s)} e^{c \int_{s}^{t} g^{2}(\tau) d \tau} d s
$$

Let us assume ${ }^{2}$ that $1 / \Lambda(s)$ is chosen such that $c\left(\Delta_{12}^{2}+\Delta_{12}^{2}\right) \leq \delta / 2$. Because $\epsilon m \in \mathcal{S}\left(\mu^{2}\right)$ implies $c \int_{s}^{t}(\epsilon(\tau) m(\tau))^{2} d \tau \leq c \mu^{2}(t-s)$, it follows that for $c\left(\Delta_{11}^{2}+\right.$ $\left.\Delta_{21}^{2}\right) \leq \delta / 2$, we have $m_{f} \in \mathcal{L}_{\infty}$. Since $m \leq m_{f}$, we have that $m \in \mathcal{L}_{\infty}$, and together with $\epsilon m \in \mathcal{L}_{2} \cap \mathcal{L}_{\infty}$ implies that $\epsilon_{1}=\epsilon m m \in \mathcal{L}_{2} \cap \mathcal{L}_{\infty}$.

We now turn our attention to the injected system (60). If we have that $T_{s d}^{*}, d_{w}=0$, the term $L(t) \epsilon_{1}$ can be viewed as an input into the exponentially stable linear system $\dot{x}=$ $A_{I}(t) x+\bar{u}$. Because $\|L(t)\| \in \mathcal{L}_{\infty}$ and $\epsilon_{1} \in \mathcal{L}_{2} \cap \mathcal{L}_{\infty}$, the input $\bar{u}=L \epsilon m^{2}$ belongs to $\mathcal{L}_{2} \cap \mathcal{L}_{\infty}$. Since $A_{I}(t)$ is e.s. and $L \epsilon_{1} \in \mathcal{L}_{2} \cap \mathcal{L}_{\infty}$, it follows from result 2) of Lemma 1 and (60) that $x \in \mathcal{L}_{2} \cap \mathcal{L}_{\infty}, \dot{x} \in \mathcal{L}_{2} \cap \mathcal{L}_{\infty}$, and $x \rightarrow 0$ as

\footnotetext{
${ }^{2}$ As in footnote 1 , this assumption can be relaxed by using the analysis approach of [2, Section 9.9.1].
}

$t \rightarrow \infty$. From the convergence of $x$, and consequently $L \epsilon_{1}$, it follows from (60) that $\dot{x} \rightarrow 0$ as $t \rightarrow 0$.

Similarly, consider that $\Delta_{m} \neq 0$ or $d_{w} \neq 0$. The dynamics of $x$ are then governed by

$$
\dot{x}=A_{I}(t) x+\bar{u}+B d_{w}
$$

where $\bar{u}=L \epsilon_{1}$ has been shown to belong to $\mathcal{S}\left(\mu^{2}\right) \cap \mathcal{L}_{\infty}$ and $d_{w}$ is bounded. Therefore, $x \in \mathcal{L}_{\infty}$, and, in turn, $\dot{x} \in$ $\mathcal{L}_{\infty}$. Moreover, from result 3) of Lemma 2, it follows that $\int_{0}^{t}|y(\tau)|^{2} d \tau \leq c\left(\Delta_{11}^{2}+\Delta_{21}^{2}+\left\|d_{w}\right\|_{\infty}^{2}\right) t+c$.

\section{CONCLUSION}

The stability and robustness properties of an AMC scheme applied to an uncertain AHFV model were analyzed, providing answers to some important theoretical questions concerning the practicality of the AMC scheme. The analysis establishes that all closed-loop states remain bounded and of the order of the modeling error provided that the complex uncertainty satisfies some bound condition. Consistent with this result, if the true aircraft dynamics matches the nominal model (i.e., in this case $T_{s d}^{*}=0$ ) the closed-loop adaptive system is asymptotically stable.

\section{ACKNOWLEDGMENTS}

This research has been supported in part by AFSOR Grant Number F49620-01-1-0489 and in part by NASA Grant No. NCC4-158 and NSF Award No. CMS-0510921. Any opinions, findings, and conclusions or recommendations expressed in this material are those of the author(s) and do not necessarily reflect the views of the National Science Foundation. National ICT Australia-NICTA is funded by the Australian Government as represented by the Department of Broadband, Communications and the Digital Economy and the Australian Research Council through the ICT Centre of Excellence program.

\section{REFERENCES}

[1] M. Kuipers, P. Ioannou, B. Fidan, and M. Mirmirani, "Robust adaptive multiple model controller design for an airbreathing hypersonic vehicle model," in Proc. AIAA Guidance, Navigation, and Control Conf., (Honolulu, Hawaii), August 2008.

[2] P. A. Ioannou and J. Sun, Robust Adaptive Control. Upper Saddle River, NJ: Prentice-Hall, 1996.

[3] P. A. Ioannou and B. Fidan, Adaptive Control Tutorial. Advances in Design and Control, SIAM, 2006.

[4] A. D. Clark, M. Mirmirani, C. Wu, S. Choi, and M. Kuipers, "An aeropropulsion integrated elastic model of a generic airbreathing hypersonic vehicle," in Proc. AIAA Guidance, Navigation, and Control Conf., AIAA Paper 2006-6560, 2006.

[5] H. Niemann, J. Stoustrup, and R. B. Abrahamsen, "Switching between multivariable controllers," Optimal Control Applications and Methods, vol. 25, pp. 51-66, 2004.

[6] I. Y. M. T. T. Tay and J. B. Moore, High Performance Control. Systems and Control: Foundations and Applications, Birkhauser, 1999.

[7] D. F. Delchamps, "Analytic feedback control and the algebraic Riccati equation," IEEE Trans. on Automatic Control, vol. 29, pp. 1031-1033, November 1986.

[8] A. S. Morse, "Towards a unified theory of parameter adaptive control: Tunability," IEEE Trans. on Automatic Control, vol. 35, pp. 1002-1012, September 1990.

[9] M. M. Polycarpou and P. A. Ioannou, "On the existence and uniqueness of solutions in adaptive control systems," IEEE Trans. on Automatic Control, vol. 38, pp. 474-479, March 1993. 